\title{
FLEXIBLE DISCRETIZATION FOR COMPUTATIONAL AEROACOUSTICS
}

\author{
M. Kaltenbacher ${ }^{1,2}$, J. Heinz ${ }^{2}$, K. Roppert ${ }^{1}$ and S. Schoder ${ }^{1}$ \\ 1 TU Graz \\ Inffeldgasse 18, $8010 \mathrm{Graz}$, Austria \\ \{manfred.kaltenbacher, klaus.roppert, stefan.schoder\}.@tugraz.at, www.igte.tugraz.at \\ 2 TU Wien \\ Getreidemarkt 9, 1060 Wien, Austria \\ johannes.heinz@tuwien.ac.at,www.mec.tuwien.ac.at
}

Key words: Flexible discretization, Non-conforming grids, Finite Elements, Computational Aeroacoustics

\begin{abstract}
This contribution discusses the capabilities of non-conforming grid techniques to allow an optimal discretization for each subdomain. In doing so, we derive the Nitsche-type mortaring formulation for the acoustic wave equation, which incorporates the physical transmission condition of continuity of acoustic pressure and its flux being the normal component of the acoustic particle velocity. The Nitschetype mortaring handles the coupling by symmetrizing the bilinear form and adding an appropriate jump term. The simulation of the edge tone demonstrates the applicability and superiority of non-conforming grids for computational aeroacoustics at low Mach numbers compared to conforming finite element methods.
\end{abstract}

\section{INTRODUCTION}

Since the beginning of computational aeroacoustics (CAA) several numerical methodologies have been proposed, and each of these methodologies tries to overcome the challenges for an effective and accurate computation of the radiated sound, which the specific problems under investigation pose. For high Reynolds and low Mach number flows two main challenges occur [1]: (1) the large disparity between the energy in the flow and the radiated acoustic energy; (2) large disparity between the size of an eddy in the turbulent flow and the wavelength of the generated acoustic sound. Hybrid schemes in CAA separate the flow from the acoustic computation using, e.g., aeroacoustic analogies. Thereby, an optimal computational grid can be used for each individual physical field achieving the highest accuracy. As a result, the two grids may be quite different according to the following criteria: (1) near walls, the flow grid needs refinement to resolve boundary layers; (2) the flow grid is mostly coarsened towards outflow boundaries to dissipate vortices; (3) the acoustic grid has to transport waves and therefore needs a uniform grid size all over the computational domain. Therefore, the numerical scheme should support flexibility in the sense of non-conforming grid techniques [2]. Thereby, each sub-domain can be meshed individually, and the numerical scheme performs the coupling over the common interfaces by fulfilling the physical transmission conditions: continuity of the primary physical quantity and its flux. Here, we 
apply Nitsche-type mortaring, which does not need the additional Lagrange multiplier and handles the coupling by symmetrizing the bilinear form and adding an appropriate jump term. The contribution will contain the fundamental steps to achieve an efficient and stable Nitsche-type mortaring formulation and a demonstrative example towards edge tone generation.

\section{Non-conforming FE formulation}

The two main approaches to allow non-conforming grids within classical FE formulations are: (1) Mortar coupling (see, e.g., [3, 4]) and (2) Nitsche-type coupling (see, e.g., [5]). The first approach guarantees the strong coupling of the numerical flux by introducing a Lagrange multiplier. Thereby the flux is the normal component of the acoustic particle velocity and can be expressed by the normal derivative of the acoustic pressure scaled by the mean density. The continuity of the acoustic pressure is treated in a weak sense. Nitsche-type coupling does not need the additional Lagrange multiplier and handles the coupling by symmetrizing the bilinear form and by adding a special jump term.

The derivation of the Nitsche-type formulation for the acoustic wave equation assumes a global domain $\Omega$ and its decomposition into two sub-domains $\Omega_{1}, \Omega_{2}$ as displayed in Fig. 1. Thus, in each sub-domain
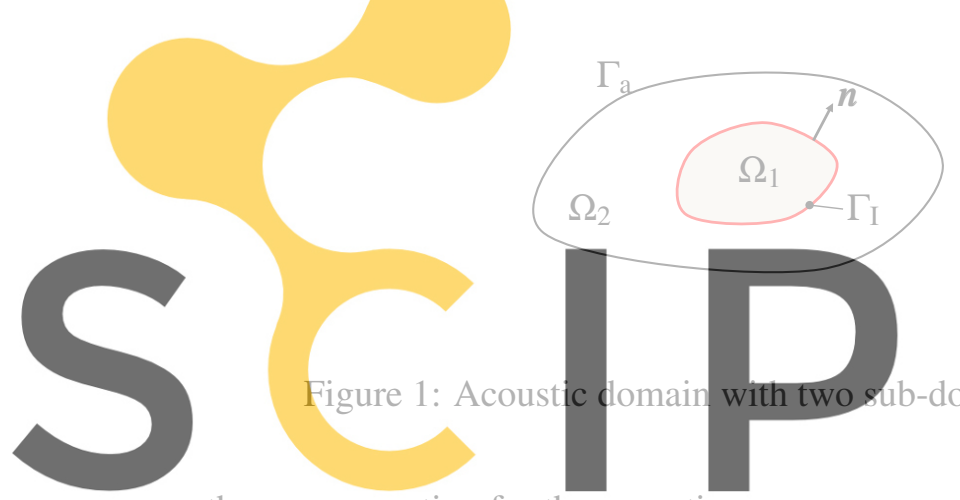

the wave equation for the acoustic pressure $p_{\mathrm{a} i}$

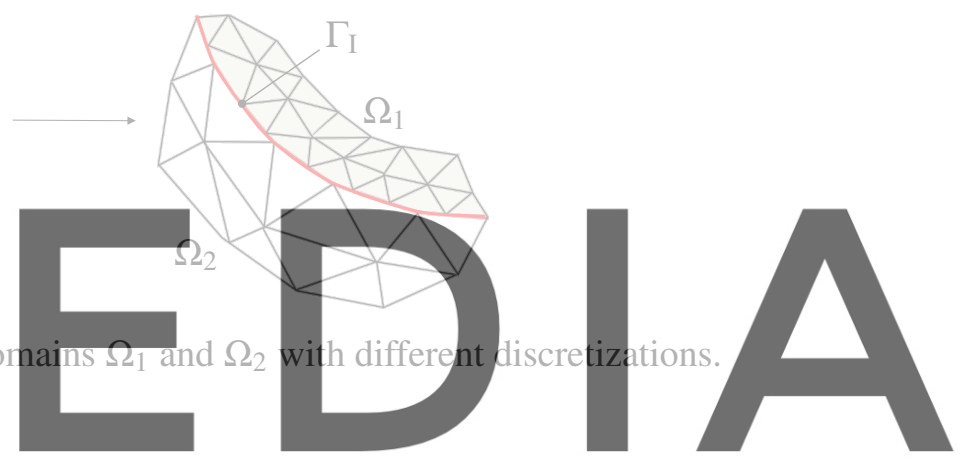

Register for free at https//www.seipedia.com to download the version without the watermark $\frac{\rho_{i} c_{i}^{2}}{\partial t^{2}}-\nabla \frac{1}{\rho_{i}} \nabla p_{\mathrm{a} i}=g_{i}, \quad$ in $\Omega_{i} \times(0, T), i=1,2$

completed by appropriate initial conditions at time $t=0$ and boundary conditions on the global boundary $\Gamma_{\mathrm{a}}$ is solved. In (1) $\rho_{i}$ denotes the mean density and $c_{i}$ the speed of sound in each subdomain $\Omega_{i}$. Both physical quantities are assumed to be constant in space and time. The physical transmission conditions along the interface $\Gamma_{I}$ impose continuity for trace and flux i.e.,

$$
p_{\mathrm{a} 1}=p_{\mathrm{a} 2} \text { and } \frac{1}{\rho_{1}} \frac{\partial p_{\mathrm{a} 1}}{\partial \boldsymbol{n}}=\frac{1}{\rho_{2}} \frac{\partial p_{\mathrm{a} 2}}{\partial \boldsymbol{n}} \text { on } \Gamma_{\mathrm{I}}
$$

with $\boldsymbol{n}$ the unit normal vector. Without any limitation and to keep the focus on the main steps achieving the Nitsche-type FE formulations, a homogeneous Dirichlet boundary condition for the acoustic pressure $p_{\mathrm{a}}$ at $\Gamma_{\mathrm{a}}$ is set.

To achieve at the non-conforming discretization within Nitsche's method, we start at the weak formula- 
tion for both sub-domains $\Omega_{1}, \Omega_{2}$ with corresponding test functions $w_{1}, w_{2}$

$$
\begin{gathered}
\int_{\Omega_{1}} \frac{1}{\rho_{1} c_{1}^{2}} w_{1} \frac{\partial^{2} p_{\mathrm{a} 1}}{\partial t^{2}} \mathrm{~d} \boldsymbol{x}+\int_{\Omega_{1}} \frac{1}{\rho_{1}} \nabla w_{1} \cdot \nabla p_{\mathrm{a} 1} \mathrm{~d} \boldsymbol{x}-\int_{\Gamma_{\mathrm{I}}} w_{1} \frac{1}{\rho_{1}} \frac{\partial p_{\mathrm{a} 1}}{\partial \boldsymbol{n}_{1}} \mathrm{~d} \boldsymbol{s}=\int_{\Omega_{1}} w_{1} g_{1} \mathrm{~d} \boldsymbol{x}, \\
\int_{\Omega_{2}} \frac{1}{\rho_{2} c_{2}^{2}} w_{2} \frac{\partial^{2} p_{\mathrm{a} 2}}{\partial t^{2}} \mathrm{~d} \boldsymbol{x}+\int_{\Omega_{2}} \frac{1}{\rho_{2}} \nabla w_{2} \cdot \nabla p_{\mathrm{a} 2} \mathrm{~d} \boldsymbol{x}-\int_{\Gamma_{\mathrm{I}}} w_{2} \frac{1}{\rho_{2}} \frac{\partial p_{\mathrm{a} 2}}{\partial \boldsymbol{n}_{2}} \mathrm{~d} \boldsymbol{s}=\int_{\Omega_{2}} w_{2} g_{2} \mathrm{~d} \boldsymbol{x} .
\end{gathered}
$$

In a next step, the two equations (3) and (4) are added and (2) is used as follows

$$
\boldsymbol{n}=\boldsymbol{n}_{1}=-\boldsymbol{n}_{2} ; \frac{1}{\rho_{1}} \frac{\partial p_{\mathrm{a} 1}}{\partial \boldsymbol{n}_{1}}=\frac{1}{\rho_{1}} \frac{\partial p_{\mathrm{a} 1}}{\partial \boldsymbol{n}}=\frac{1}{\rho_{2}} \frac{\partial p_{\mathrm{a} 2}}{\partial \boldsymbol{n}}=-\frac{1}{\rho_{2}} \frac{\partial p_{\mathrm{a} 2}}{\partial \boldsymbol{n}_{2}} .
$$

These steps result in

$$
\begin{array}{r}
\int_{\Omega_{1}} \frac{1}{\rho_{1} c_{1}^{2}} w_{1} \frac{\partial^{2} p_{\mathrm{a} 1}}{\partial t^{2}} \mathrm{~d} \boldsymbol{x}+\int_{\Omega_{1}} \frac{1}{\rho_{1}} \nabla w_{1} \cdot \nabla p_{\mathrm{a} 1} \mathrm{~d} \boldsymbol{x}+\int_{\Omega_{2}} \frac{1}{\rho_{2} c_{2}^{2}} w_{2} \frac{\partial^{2} p_{\mathrm{a} 2}}{\partial t^{2}} \mathrm{~d} \boldsymbol{x}+\int_{\Omega_{2}} \frac{1}{\rho_{2}} \nabla w_{2} \cdot \nabla p_{\mathrm{a} 2} \mathrm{~d} \boldsymbol{x} \\
-\int_{\Gamma_{1}}[w] \frac{1}{\rho_{1}} \frac{\partial p_{\mathrm{a} 1}}{\partial n} \mathrm{~d} s=\int_{\Omega_{1}} w_{1} g_{1} \mathrm{~d} x+\int_{\Omega_{2}} w_{2} g_{2} \mathrm{~d} x .
\end{array}
$$

In (5) the operator [ ] defines the jump operator, e.g., $[w]=w_{1}-w_{2}$. In order to retain symmetry, the following term is added to (5)
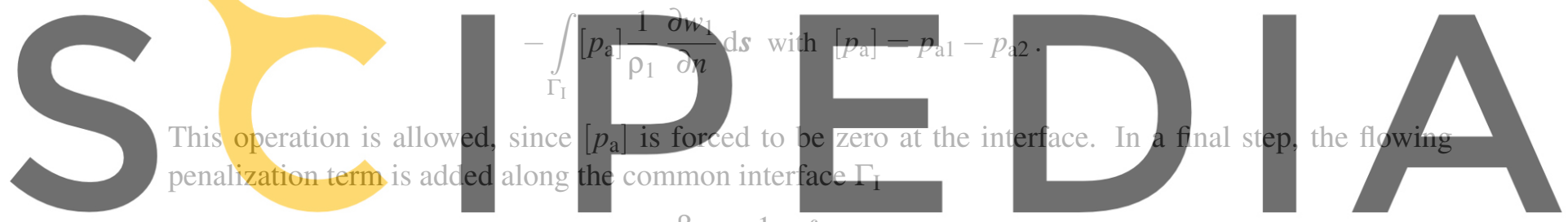

\section{$\beta \frac{1}{h_{F}} \int\left[p_{\mathrm{a}}\right][w] \mathrm{d} \mathrm{d}$}

(6)

Register for free at https//www.scipedia?.com $\mathrm{EQ}_{\mathrm{E}} \mathrm{download}$ the version without the watermark

where $\partial \Omega^{(e)}$ is the boundary of an element and $\Gamma_{E}$ is the element boundary along the non-conforming interface $\Gamma_{E}=\partial \Omega^{(e)} \cap \Gamma_{I}$ and $\beta$ the penalty factor. In (6) $h_{E}$ is a characteristic length scale of each interface element $\mathrm{E}$ (space discrete level). Therewith, we arrive at the following final formulation for Nitsche-type mortaring

$$
\begin{array}{r}
\int_{\Omega_{1}} \frac{1}{\rho_{1} c_{1}^{2}} w_{1} \frac{\partial^{2} p_{\mathrm{a} 1}}{\partial t^{2}} \mathrm{~d} \boldsymbol{x}+\int_{\Omega_{1}} \frac{1}{\rho_{1}} \nabla w_{1} \cdot \nabla p_{\mathrm{a} 1} \mathrm{~d} \boldsymbol{x}+\int_{\Omega_{2}} \frac{1}{\rho_{2} c_{2}^{2}} w_{2} \frac{\partial^{2} p_{\mathrm{a} 2}}{\partial t^{2}} \mathrm{~d} \boldsymbol{x} \\
+\int_{\Omega_{2}} \frac{1}{\rho_{2}} \nabla w_{2} \cdot \nabla p_{\mathrm{a} 2} \mathrm{~d} \boldsymbol{x}-\int_{\Gamma_{\Gamma_{1}}[w] \frac{1}{\rho_{1}} \frac{\partial p_{\mathrm{a} 1}}{\partial n} \mathrm{~d} \boldsymbol{s}}-\int_{\text {Consistency }}^{\int_{\Gamma_{1}}\left[p_{\mathrm{a}}\right] \frac{1}{\rho_{1}} \frac{\partial w_{1}}{\partial n} \mathrm{~d} \boldsymbol{s}} \\
+\underbrace{\frac{\beta}{\rho_{1}} \sum_{E} \frac{1}{h_{E}} \int_{\Gamma_{E}}\left[p_{\mathrm{a}}\right][w] \mathrm{d} \boldsymbol{s}}_{\text {Symmetrization }}=\int_{\Omega_{1}} w_{1} g_{1} \mathrm{~d} \boldsymbol{x}+\int_{\Omega_{2}} w_{2} g_{2} \mathrm{~d} \boldsymbol{x} .
\end{array}
$$


If the penalty parameter $\beta$ is chosen large enough, the bilinear form is coercive on the discrete space and one derives optimal a priori error estimates in both the energy norm and the $L_{2}$ norm for polynomials of arbitrary degree [5]. As analyzed in [6], factor $\beta$ should be chosen approximately as 20 to achieve a minimal numerical error. The space discretization can be performed with standard Lagrangian basis functions (for details, see, e.g., [7]). For the computation of the matrix entries coming from the surface integrals along the common interface $\Gamma_{\mathrm{I}}$, grid intersection operations are necessary. A detailed discussion on this topic can be found in [8] include both plane and curved interfaces. The time discretization results in an algebraic system of equations to be solved for each time step. Here, a Hilber-Hughes-Taylor time discretization is applied which allows controlled numerical dispersion (for details, we refer to [6]).

Finally, we want to note that Nitsche-type mortaring is equivalent to an IP-DG (Internal Penalty - Discontinuous Galerkin) ansatz being applied along the non-conforming interface $\Gamma_{\mathrm{I}}$.

\section{Application: Edge tone}

Edge tones can be encountered in several areas. Musical woodwind instruments like a flute or an organ pipe all share the principle of the sound generation by an air jet which oscillates around a wedgeshaped object. In addition to this sound generation mechanism, the mentioned instruments exhibit also an attached Helmholtz resonator for the amplification of the generated sound. In general however, no resonator is required for the generation of sound. The air jet oscillates with relatively stable frequencies
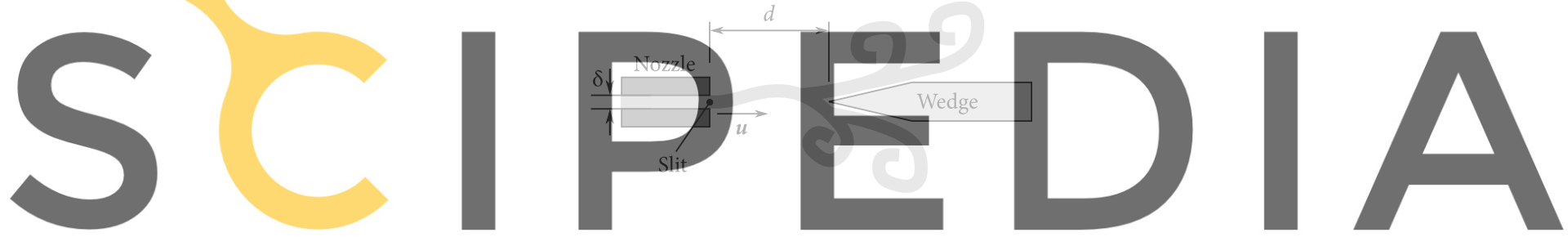

Figure 2: Schematic of edge tone configuration.

\section{Register for free at https//www.scipedia.com to download the version without the watermark}

which depend on the distance $d$ of the wedge to the slit of the nozzle, the mean inflow velocity $u_{\text {mean, }}$, the

viscosity of the medium $v_{f}$ and the inflow profile of the jet (see Fig. 2). Under certain circumstances, audible tones are emitted. By varying the inflow velocities or the slit-edge distance, the emitted frequencies exhibit regions with continuous changes but there exist also jumps in the frequency. The continuous regions between jumps are referred to as stages of the edge tone and are assigned ordinal numbers. The first stage of an edge tone corresponds to a situation where there is one half-wave present in the shape of the jet between slit and edge. Higher stages are defined correspondingly.

\subsection{Problem Description}

The edge tone is numerically most demanding and demonstrates the powerful applicability of nonconforming meshes, because the whole CFD domain fits into one element of the acoustic propagation mesh at the main frequency of $130 \mathrm{~Hz}$. The geometrical setup for the computational CFD domain can be seen in Fig. 3, where we just display half models due to the geometric symmetry. The Reynolds number 


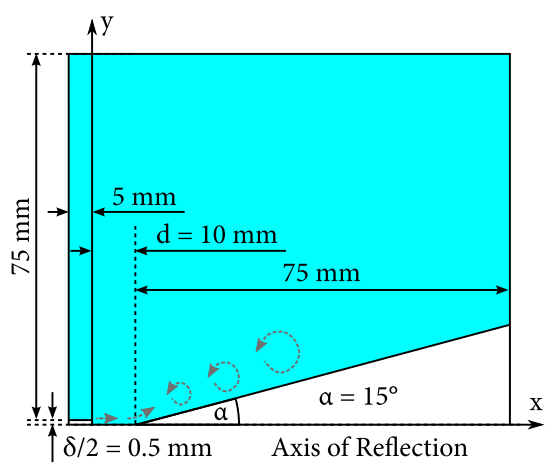

(a) View from top.

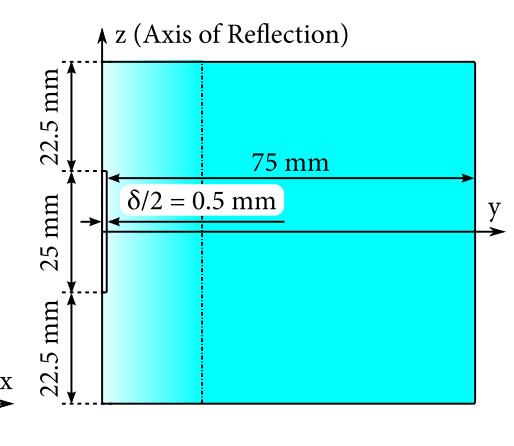

(b) View from front.

Figure 3: CFD domain setup for edge tone.

computes by

$$
\operatorname{Re}=\frac{u \delta}{v_{\mathrm{f}}}=225,
$$

where $\delta=1 \mathrm{~mm}$ is the slit width of the nozzle and $u$ the characteristic velocity, e.g. the inlet velocity.

The applied physical properties and boundary conditions are as follows:

- Material properties

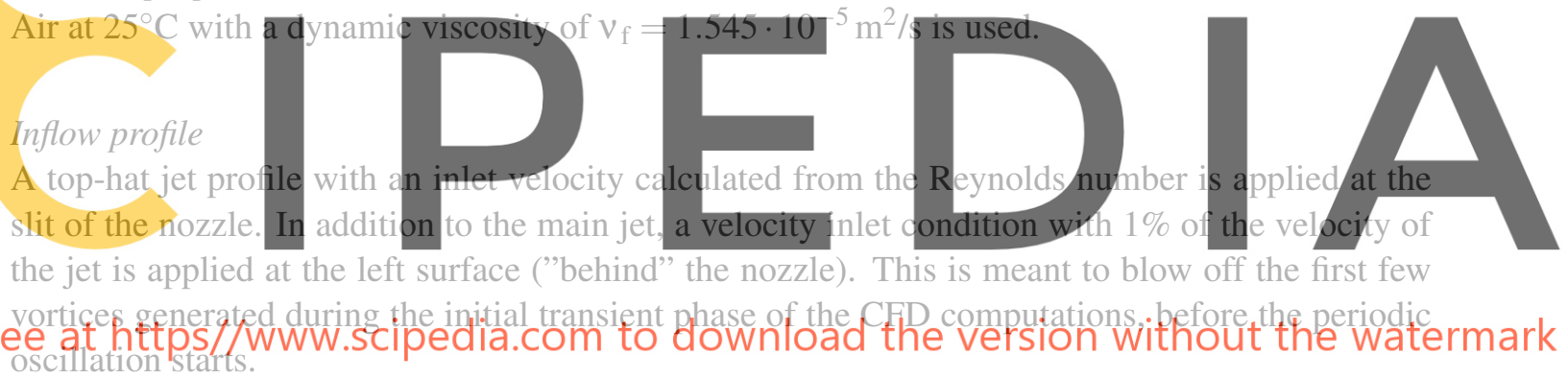

- Boundary conditions

A free slip wall condition is applied at the nozzle wall to avoid vortex generation. On the wedge wall a no slip condition is applied. On all other surfaces opening conditions are used.

\section{- Time stepping}

The time step is $170 \mu$ s and the computed incompressible velocity field is written out to an output file every second step.

\section{- Turbulence model}

No turbulence model is applied and the flow is treated as laminar and incompressible. 


\section{- Input for the acoustic simulation}

The transient simulation is run until the oscillation of the jet reaches a stationary state. Beginning from the corresponding time step, 300 transient output steps, corresponding to a time interval of $102 \mathrm{~ms}$ length (13 stage 1 oscillations), are used for the acoustic investigations. The transient sources are then interpolated to different acoustic grids of the source region and a FFT is performed to obtain harmonic sources at the main frequency of $130 \mathrm{~Hz}$.

The spatial discretization for the CFD computation in the vicinity of the nozzle is depicted in Fig. 4a. The mesh is symmetric to the $\mathrm{x}-\mathrm{z}$-plane and the smallest edge-length (corresponding to the discretization of the wedge-wall-boundary layer) is about $5 \mu \mathrm{m}$ whereas the coarsest one is about $5 \mathrm{~mm}$. The mesh solely consists of hexahedra and the number of nodes is 715.268. Computations with an unstructured and unsymmetrical CFD mesh (cf. Fig. 4b), which also contained wedge elements, resulted in unsymmetrical dipole characteristics in the acoustic simulations. The computational domain for acoustics just consists of

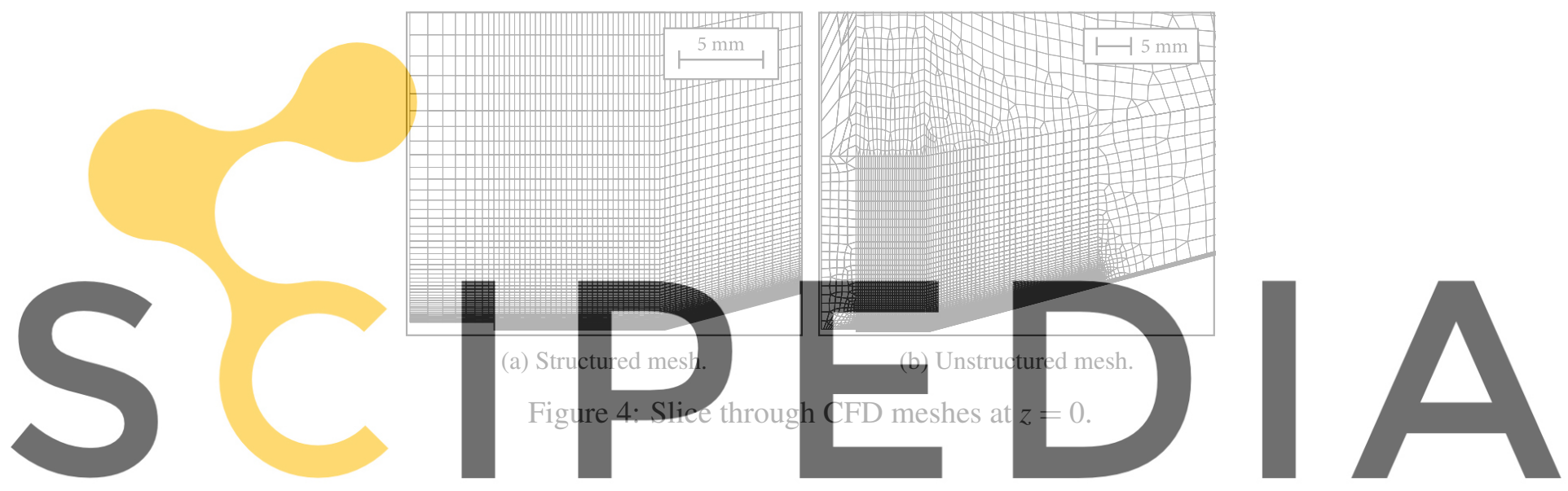

the source domain $\Omega_{\mathrm{S}}$ and three cube-shaped domains $\Omega_{\mathrm{i}}, \Omega_{\mathrm{p}}, \Omega_{\mathrm{PML}}$ of different sizes which are centered

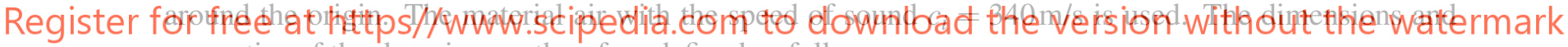
properties of the domains are therefore defined as follows:

- Source domain

Unlike in the CFD mesh, the nozzle volume is completely discretized by volume elements. Otherwise the geometry of the source domain is the same as in Fig. 3.

- Intermediate domain

Since elements in the propagation mesh can be bigger than the whole source domain an intermediate domain for bridging the gap between the propagation mesh and the source mesh with a structured mesh is introduced. The intermediate mesh has non-matching interfaces with $\Omega_{\mathrm{s}}$ as well as with $\Omega_{\mathrm{p}}$. With the variable parameter $w$ it is defined as

$$
\Omega_{\mathrm{i}}=[-w \ldots w]^{3} \backslash \Omega_{\mathrm{s}} .
$$

\section{- Propagation domain}

The acoustic propagation domain is discretized with a structured mesh corresponding to the main frequency component of $130 \mathrm{~Hz}$. The elements in this domain have the same edge length in all 
three dimensions. The domain is defined as

$$
\Omega_{\mathrm{p}}=[-3 \mathrm{~m} \ldots 3 \mathrm{~m}]^{3} \backslash\left(\Omega_{\mathrm{s}} \cup \Omega_{\mathrm{i}}\right) .
$$

\section{- Damping Layer}

In order to simulate free-field conditions an additional perfectly matched layer (PML) is attached to the computational domain [9]. Its thickness of $80 \mathrm{~cm}$ nearly corresponds to one third of the wave length $\lambda=2.62 \mathrm{~m}$ at $130 \mathrm{~Hz}$. The discretization from $\Omega_{\mathrm{p}}$ is carried on conforming across the domain boundary. The PML domain is defined as

$$
\Omega_{\mathrm{PML}}=[-3.8 \mathrm{~m} \ldots 3.8 \mathrm{~m}]^{3} \backslash\left(\Omega_{\mathrm{s}} \cup \Omega_{\mathrm{i}} \cup \Omega_{\mathrm{p}}\right) .
$$

For the acoustic computation performed by openCFS [11], Lighthill's aeroacoustic analogy for low Mach numbers is applied (for details, we refer to [10]). In doing so, the source term fitting to the wave equation (1) and assuming just sources in the subdomain $\Omega_{1}$ computes by

$$
g_{1}=\frac{\partial^{2} L_{i j}}{\partial x_{i} \partial x_{j}} ; \quad L_{i j}=u_{i}^{\text {ic }} u_{j}^{\text {ic }}
$$

with $u_{i}$ the $i$-th component of the incopressible flow velocity. This source term is evaluated at the CFD mesh and then conservatively interpolated to the acoustic grid [12]

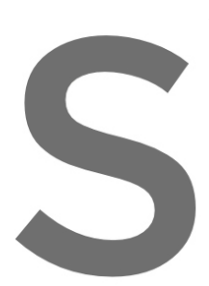

With the given geometrica

tivity of the edge tone cor

- ETFINE: This case

in the source region

Tri-linear hexahedr
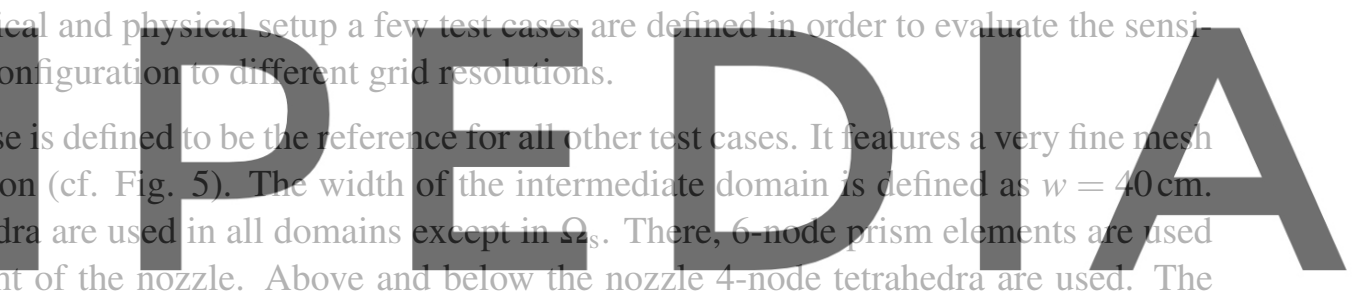

coarsest edge length in this mesh is about $6.66 \mathrm{~cm}$ which corresponds to a minimal value of about

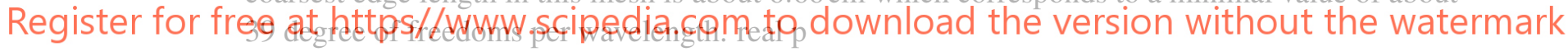

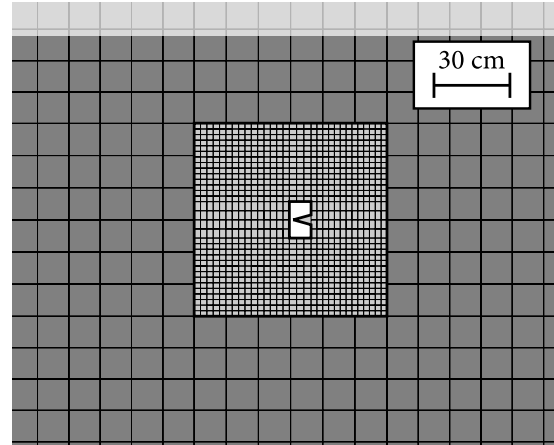

Figure 5: Cut through intermediate and propagation meshes at $z=0$ for ETFINE case.

- ETCOFA: The same fine source mesh as in the ETFINE case is extended with a very coarse mesh in $\Omega_{\mathrm{p}}$. The edge length of $26.66 \mathrm{~cm}$ still guarantees a resolution of about 10 degree of freedoms 
per wavelength. Since the whole source domain fits into one element of $\Omega_{\mathrm{p}}$ (depicted by the outer dashed white line in Fig. 6a), the width of the intermediate mesh $w=13.33 \mathrm{~cm}$ in $\Omega_{\mathrm{i}}$ is chosen in a way so that it just bridges this gap. In all domains except $\Omega_{\mathrm{s}}$ linear hexahedra are used.

- ETCONE: The same mesh for the outer domains as in the ETCOFA case is used. The difference is however, that quadratic serendipity hexahedra are used, which account for about 20 degree of freedoms per wavelength. In the source domain $\Omega_{\mathrm{s}}$ a coarser unstructured mesh is applied compared to the previous cases (cf. Fig. 6b).

real $\mathrm{p}$
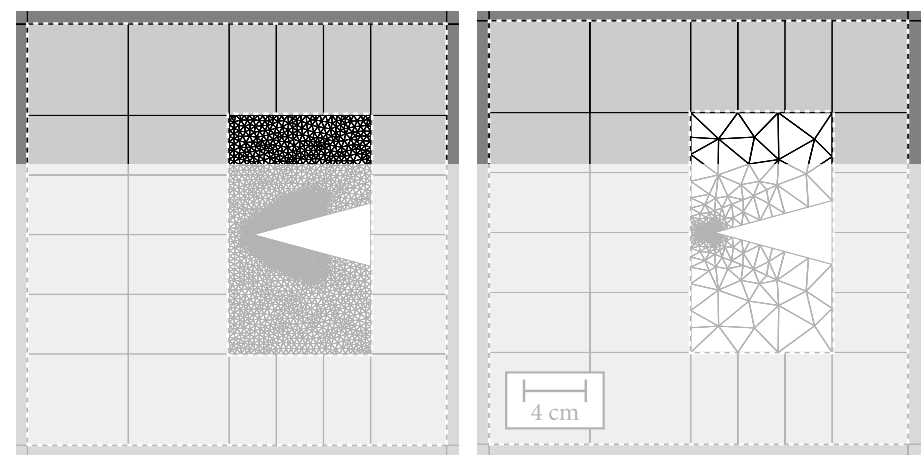

(a) Meshes for ETCOFA case.

(b) Meshes for ETCONE case.
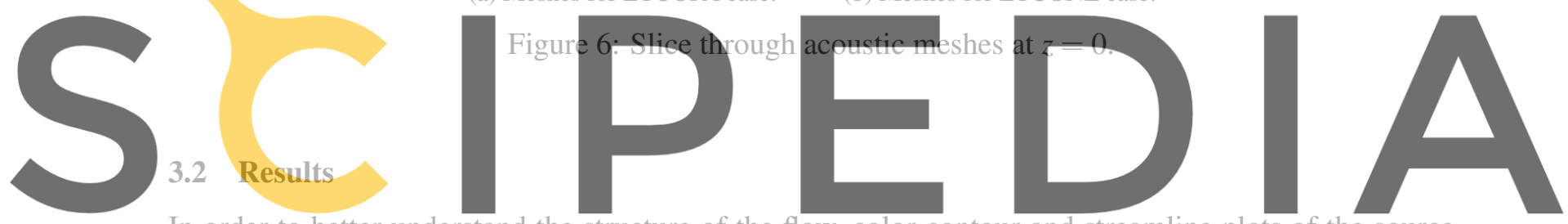

In order to better understand the structure of the flow, color-contour and streamline plots of the source

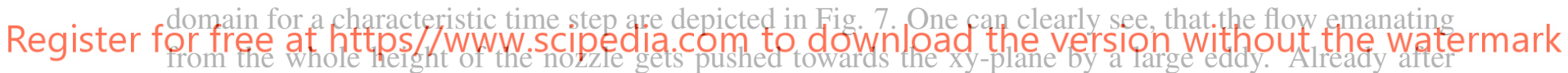
about one third of the length of the wedge, the flow is mainly concentrated around the xy-plane. After the interpolation to the dedicated acoustic grid, the same behavior can also be observed for the harmonic Lighthill source term at the main frequency of $130 \mathrm{~Hz}$ (cf. Fig. 8). A cut through the source domain mesh of the ETFINE and the ETCOFA cases is shown. In addition to that, isocontours and a color mapped plot at $z=0$ of the real part of the acoustic source terms are shown.

The acoustic computations show the expected dipole field for the whole acoustic domain as displayed in Fig. 9, where the real part of the acoustic pressure field at $130 \mathrm{~Hz}$ for ETFINE case is depicted. The shown isocontours of the acoustic pressure have been evaluated at $\pm 50 \mu \mathrm{Pa}$. A comparison of the directivity patterns of the sound pressure levels in the xy- and yz-planes is depicted in Fig. 10. It reveals that coarser meshes, either in the source or the propagation domain, tend to under-predict the sound pressure levels. However, the maximal deviation is in the range of $1 \mathrm{~dB}$. Again considering the fact, that the whole source domain is enclosed in a single element of the far-field domain, the obtained results match the reference case ETFINE quite well. The number of unknowns for the different computational meshes are listed in Tab. 1. 


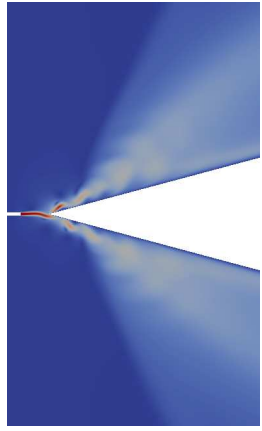

(a) Fluid velocity field at $z=0$

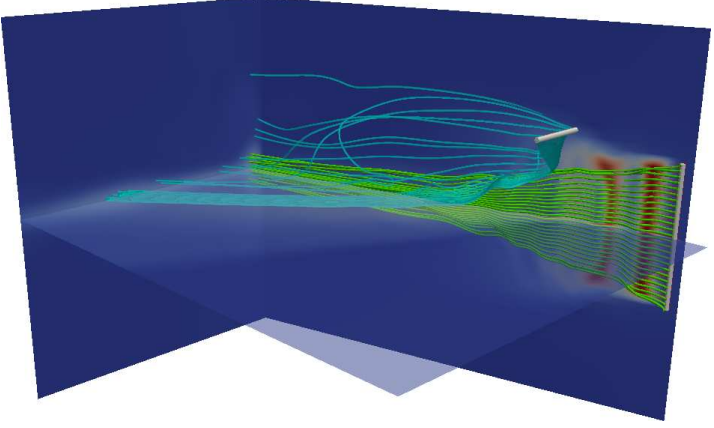

(b) Stream lines

Figure 7: Structure of the fluid velocity field.

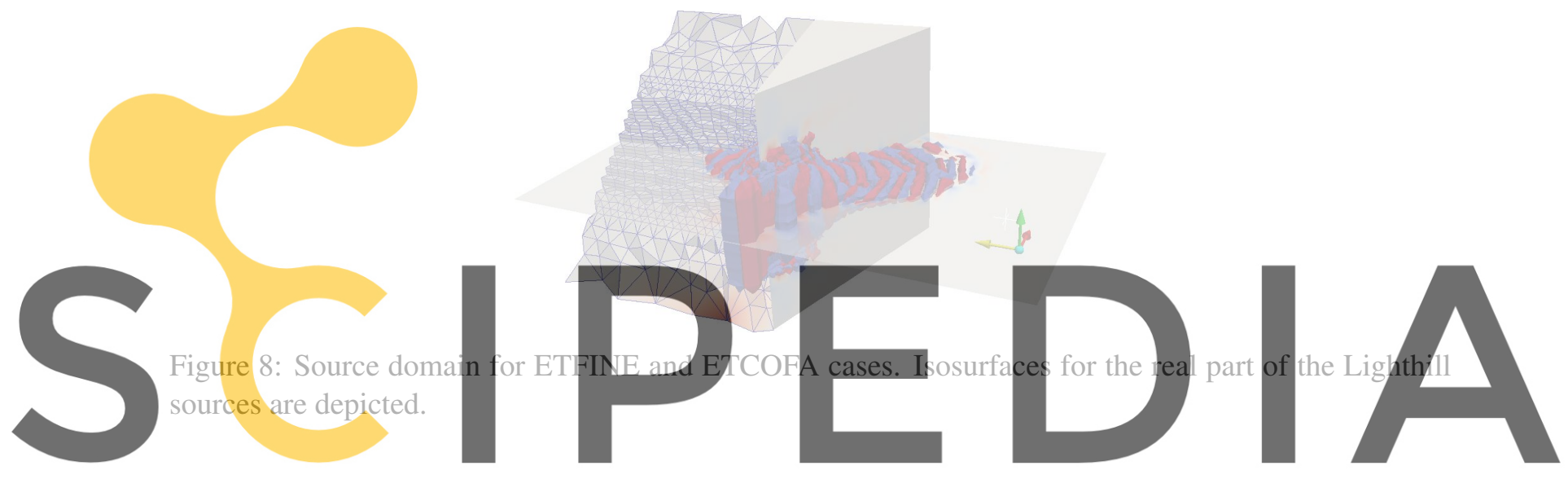

4 CONCLUSIONS

Register for free at https/dwww scipedia,com to download the version without the watermark

plied to the computation of the edge tone. Thereby, the physical transmission conditions of continu-

ity of acoustic pressure and its flux are used and the formulation preserves symmetry. In low Mach number aeroacoustics, where large scale disparities between flow structures and acoustic waves occur, non-conforming grid techniques demonstrate their applicability and superiority compared to conforming FE formulations. Despite full DG formulations, such an approach just needs the DG ansatz along non-conforming grid interfaces.

Table 1: Number of unknowns for the different domains.

\begin{tabular}{l|r|r|r} 
Test case & $\Omega_{\mathrm{s}}$ & $\Omega_{\mathrm{i}}$ & $\Omega_{\mathrm{p}} \cup \Omega_{\mathrm{PML}}$ \\
\hline ETFINE & 144,788 & 35,892 & 217,952 \\
ETCOFA & 144,788 & 380 & 30,176 \\
ETCONE & 11,905 & 1,343 & 114,824
\end{tabular}




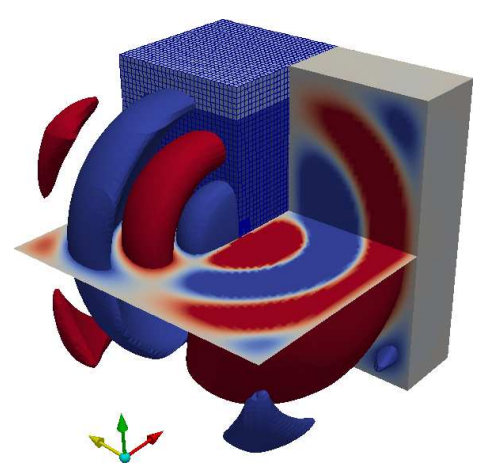

Figure 9: Acoustic pressure plot over complete domain.

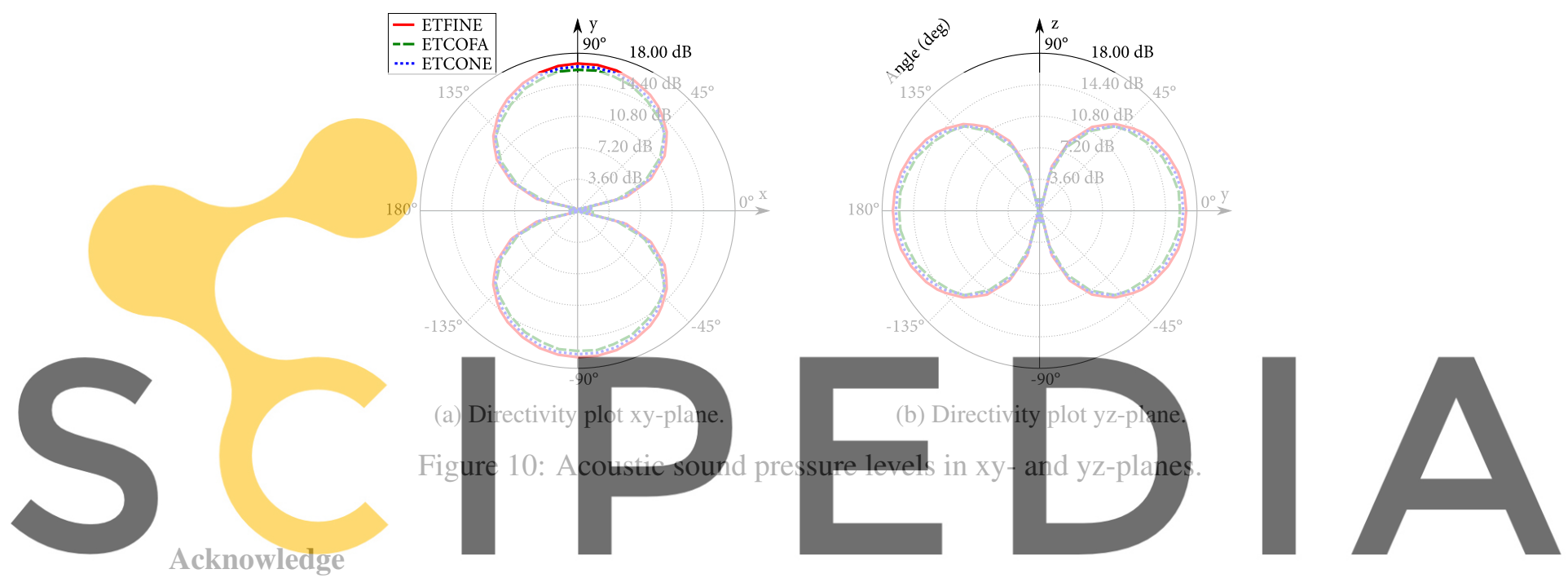

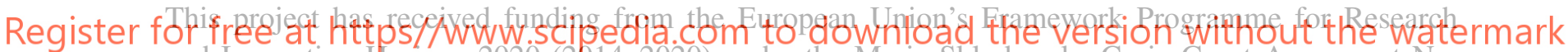 and Innovation Horizon 2020 (2014-2020) under the Marie Skłodowska-Curie Grant Agreement No. [812719].}

\section{REFERENCES}

[1] Wagner, C., Hüttl, T., Sagaut, P. (Eds.). Large-Eddy Simulation for Acoustics. Cambridge University Press, (2007)

[2] Annavarapu, C., Hautefeuille, M., Dolbow, J.E. A robust Nitsche's formulation for interface problems. Comput. Methods Appl. Mech. Engrg., (2012), 225-228: 44-54

[3] Bernardi, C., Maday, Y., Patera, A. T. (1994). A new nonconforming approach to domain decomposition: The mortar element method. Nonlinear partial differential equations and their applications. College de France Seminar, (1991), 299: 13-51

[4] Wohlmuth, B. I. A mortar finite element method using dual spaces for the Lagrange multiplier. SIAM Journal on Numerical Analysis, (2000), 38(3):989-1012

[5] Hansbo, A., Hansbo, P., Larson, M. G. A finite element method on composite grids based 
on Nitsche's method. ESAIM: Mathematical Modelling and Numerical Analysis, (2003), 37(3): 495-514

[6] Kaltenbacher, M., Hüppe, A., Grabinger, J., and Wohlmuth, B. Modeling and Finite Element Formulation for Acoustic Problems Including Rotating Domains. AIAA Journal, (2016), 54(12): 3768-3777.

[7] Kaltenbacher, M. Numerical Simulation of Mechatronic Sensors and Actuators: Finite Elements for Multiphysics, 3rd ed., Springer, (2015)

[8] Triebenbacher, S. Nonmatching Grids for the Numerical Simulation of Problems from Aeroacoustics and Vibroacoustics, PhD thesis, Alps Adriatic University Klagenfurt, Austria, (2019)

[9] Kaltenbacher, B., Kaltenbacher, M., and Sim, I. A Modified and Stable Version of a Perfectly Matched Layer Technique for the 3-D Second Order Wave Equation in Time Domain with an Application to Aeroacoustics, Journal of Computational Physics, (2013), 235: 407-422.

[10] Schoder, S., Kaltenbacher, M. Hybrid Aeroacoustic Computations: State of Art and New Achievements, Journal of Theoretical and Computational Acoustics, (2020), 27(4)

[11] openCFS - Open Source Finite Element Software for multiphysical simulation. https://opencfs.org/, 2020. Accessed: 2021-01-27.

[12] Schoder, S., Roppert, K., Weitz, M., Junger, C., Kaltenbacher, M. Aeroacoustic source term computation based on radial basis functions, International Journal for Numerical Methods in Engineering, (2019), 121(9):2051-2067 